\title{
Insight into Two-Dimensional Borophene: Five-Center Bond and Phonon-Mediated Superconductivity
}

\author{
Zhibin Gao, ${ }^{, \dagger}$ Mengyang Li, ${ }^{\ddagger}$ and Jian-Sheng Wang ${ }^{\dagger}$ \\ $\dagger$ Department of Physics, National University of Singapore, Singapore 117551, Republic of \\ Singapore \\ $\ddagger$ Institute for Chemical Physics \& Department of Chemistry, Graduate Schoolof Science, \\ Xi'anJiaotong University, Xi'an 710049, China \\ E-mail: zhibin.gao@nus.edu.sg
}

\section{Abstract}

We report a previously unknown monolayer borophene allotrope and we call it super-B with a flat structure based on the ab initio calculations. It has good thermal, dynamical, and mechanical stability compared with many other typical borophenes. We find that super-B has a fascinating chemical bond environment consisting of standard $s p, s p^{2}$ hybridizations and delocalized five-center three-electron $\pi$ bond, called $\pi(5 \mathrm{c}-3 \mathrm{e})$. This particular electronic structure plays a pivotal role in stabilizing the super-B chemically. By extra doping, super-B can be transformed into a Dirac material from pristine metal. Like graphene, it can also sustain tensile strain smaller than $24 \%$, indicating superior flexibility. Moreover, due to the small atomic mass and large density of states at the Fermi level, super-B has the highest critical temperature $T_{c}$ of $25.3 \mathrm{~K}$ in single-element superconductors at ambient condition. We attribute this high $T_{c}$ of super-B to the giant anharmonicity of two linear acoustic phonon branches and an unusually low optic phonon mode. These predictions provide new insight into the chemical nature of low dimensional boron nanostructures and highlight the potential applications of designing flexible devices and high $T_{c}$ superconductor.

\section{Keywords}

Ab initio calculations, Dirac cone, electronic structure, charge doping, strain effect, superconductivity, electron-phonon coupling, 2D boron

\section{Introduction}

Boron atom has five electrons with an electron configuration $1 \mathrm{~s}^{2} 2 \mathrm{~s}^{2} 2 \mathrm{p}^{1}$, which means that the number of valence electrons is less than the available orbitals. Due to the small atomic radius, removal of valence electrons of boron requires a large amount of energy. Therefore, boron likely forms covalent compounds, rather than $\mathrm{B}^{3+}$ ions. However, owing to the smaller electronegativity than hydrogen, boron atoms have a little positive charge in most covalent compounds, except for special B-B bonds.

Matter always wants to be in the most stable form. For most of non-metal atoms, stability is achieved by following the octet rule. Theoretically, boron can accommodate five more electrons according to the octet rule, but boron commonly emerges three bonds, like $\mathrm{BH}_{3}$, with a total of six electrons in the outermost shell excepting for coordination compounds with four ligands like $\left[\mathrm{BF}_{4}\right]^{-}$and $\mathrm{B}[\mathrm{OH}]_{4}^{-}$. Hence, con- 
ventional $2 \mathrm{c}-2 \mathrm{e}$ bond does not hold in the "electron-deficiency" boron compounds. ${ }^{1-3}$ Afterwards, many researchers find that localized three-centered bonds, multi-centered bonds, and delocalized $\sigma$ and $\pi$ bonds play very crucial roles in stabilizing the boron compounds. ${ }^{4,5}$ This particular electronic configuration also results in many anomalous properties in boron compounds. $^{1,6,7}$

In 2012, for the first time, polymorphism of $2 \mathrm{D}$ boron, called borophene, was proposed ${ }^{8}$ and proper substrates were further explored theoretically, ${ }^{9,10}$ which play pivotal roles in finally synthesized borophene experimentally. ${ }^{11,12}$

In this study, we predict a previously unknown 2D borophene allotrope, called super-B due to the large vacancy, based on the ab initio calculations. Super-B is a hexagonal borophene with three atoms in each side, different from the hexagonal graphene. Besides, it has good thermal, dynamical, and mechanical stability. We find that the structure of super-B is formed by the standard $s p, s p^{2}$ and delocalized $\pi(5 c-$ $3 \mathrm{e})$ bonds in terms of the natural bond orbital (NBO) analysis. These particular type of chemical bonds have never been reported in borophene before. Therefore, super-B sets a good example to break the stereotype that triangular lattice with hexagonal vacancies is the only principle and concept of borophene. Furthermore, by doping method, super-B can be transformed into a Dirac material from pristine metal. It can also sustain $24 \%$ of biaxial strain before fracture, indicating the same superior flexibility as graphene. Due to the small atomic mass and large density of states at Fermi level, super-B has a phonon-mediated superconducting $T_{c}$ of $25.3 \mathrm{~K}$, which is derived from the giant anharmonicity of two linear acoustic phonon branches and an unusually low optic $O_{z}$ phonon mode.

\section{Computational details}

We studied the electronic structure, the equilibrium geometry, and structural stability of super-B using ab initio DFT as implemented in VASP. ${ }^{13-15}$ We used the exchange-correlation functional of Perdew-Burke-Ernzerhof $(\mathrm{PBE})^{16}$ and HSE06 ${ }^{17,18}$ with default mixing parameter value $\alpha=0.25$. The vacuum distance over adjacent boron layer is set to be $20 \AA$. The plane-wave cutoff is set to $500 \mathrm{eV}$. For structure optimization, both lattice constants and atomic positions are relaxed with the criterion for total energy of $1.0 \times 10^{-8} \mathrm{eV}$ and Hellmann-Feynman forces of $10^{-4} \mathrm{eV} / \AA$. The Brilliouin zone is sampled by $11 \times 11$. For the electron-phonon coupling (EPC), we used Quantum Espresso ${ }^{19}$ with 80 Ry energy cutoff. The self-consistent electron density in super-B was calculated by a $64 \times 64 \mathrm{k}$-point grid. The dynamical matrices were calculated on a $10 \times$ 10 grid after convergence test. The NBO analysis was carried out on B3LYP/6-311G(d,p) with Gaussian $16^{20}$ after optimization of B3LYP/6$31 \mathrm{G}(\mathrm{d}, \mathrm{p})$.

\section{Results}

\section{Structure}

The optimized super-B is shown in Figure 1a. The lattice constants are $\left|\overrightarrow{a_{1}}\right|=\left|\overrightarrow{a_{2}}\right|=5.45 \AA$ with $P 6 / \mathrm{mmm}$ (space group no. 191). There are two independent atoms based on the group Wyckoff positions, whose fractional coordinates are $\left(\frac{1}{3}, \frac{2}{3}\right)$ and $\left(\frac{1}{2}, \frac{1}{2}\right)$. In order to perform the chemical bond analysis quantitatively, we use hydrogen atoms to saturate boron atoms at the border and explore the primitive cell located in the center region marked by a red box with negligible fringe effect. Each boron atom is labelled by a subscript number. We find that $\mathrm{B}_{3}$ is coordinated with three $\mathrm{B}\left(\mathrm{B}_{1}, \mathrm{~B}_{2}\right.$ and $\left.\mathrm{B}_{4}\right)$ via $s p^{2}$ hybridization, and $\mathrm{B}_{4}$ is linked to the $\mathrm{B}_{3}$ and $\mathrm{B}_{5}$ with $s p$ hybridization. The detailed NBO analysis is shown in Table 1 . It is clear that each boron atom has a $p$ orbital which is perpendicular to the plane of super$\mathrm{B}$ in Figure 1b, and these $p$ orbitals of boron, unhybridized and perpendicular to the plane of super-B, constitute an electron-deficiency $\pi(5 \mathrm{c}-$ $3 \mathrm{e})$ bond in the primitive cell, which is similar to the $\pi(2 \mathrm{c}-2 \mathrm{e})$ orbital in the primitive cell of graphene. Therefore, on the basis of period- 
(a)

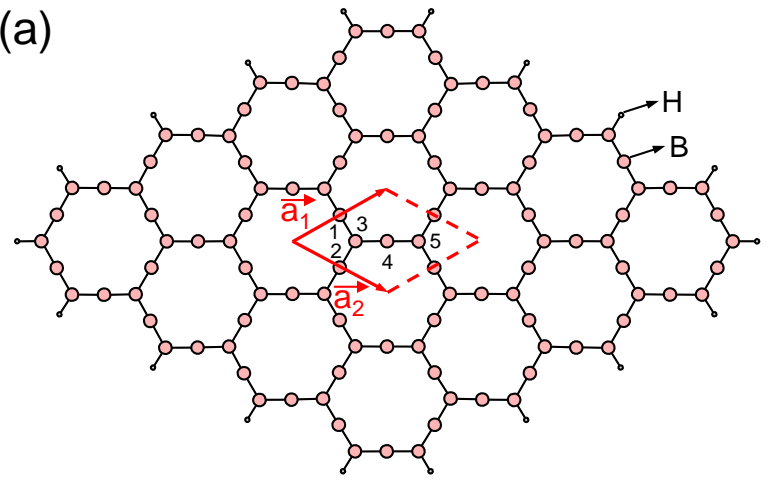

(c)

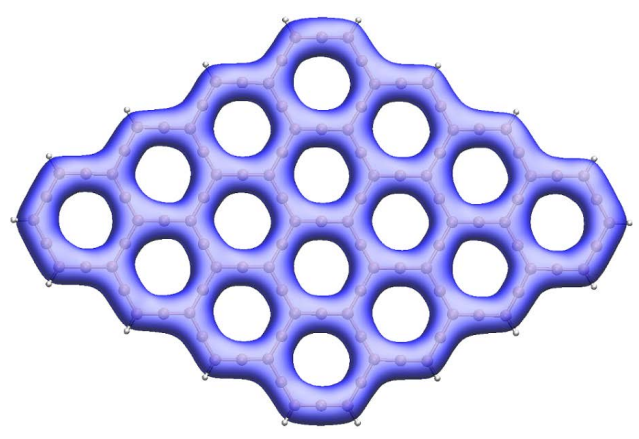

(b)

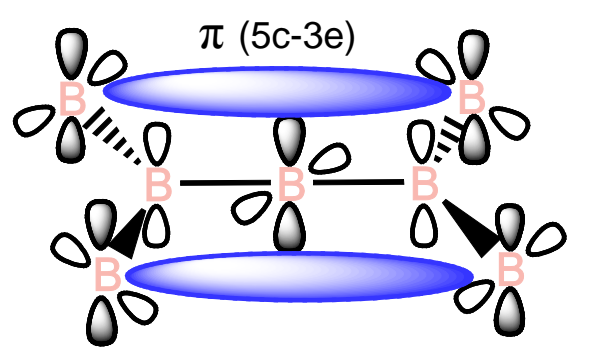

(d)

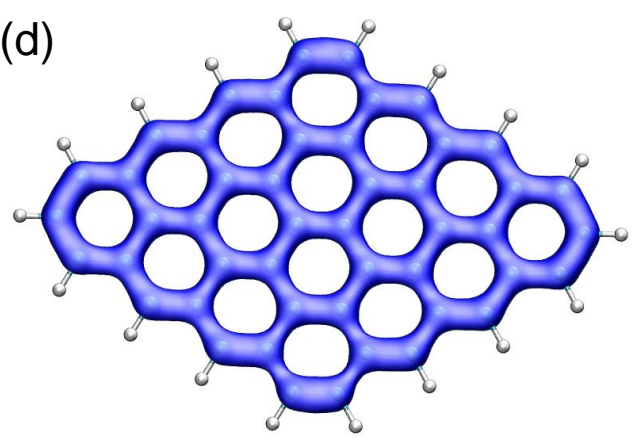

Figure 1: (a) Crystal structure of super-borophene (super-B). The marginal boron atoms are saturated by hydrogens and the primitive cell is denoted by a red box. Each boron atom is labelled by a cardinal number. (b) The hybridized orbital analysis in the primitive cell. Dotted and solid wedge shapes represent the boron atoms perpendicular to the plane of paper outside and inside respectively, which means seven boron atoms stand in same plane. One $p$ orbital of each boron atom, totally seven, is perpendicular to plane of super-B (parallel to plane of paper), which is shown by two shaded dumbbells with one electron for $s p$ and two white dumbbells without electron for $s p^{2}$ hybridization. All delocalized orbitals perpendicular to the plane of super-B construct the large $\pi(5 \mathrm{c}-3 \mathrm{e})$ bond. The other $p$ orbitals of $s p$ boron atoms is parallel to the plane of super-B shown by two white dumbbells. Localized orbital locator (LOL) maps for (c) super-B and (d) graphene, showing strong $\pi$ bonds (isovalue $=0.4$ a.u.).

icity rule, a large delocalized $\pi(5 n c-3 n e)$ bond with electron-deficiency characteristic emerges on the surface of super-B.

In order to further verify the interesting $\pi$ bond, localized orbital locator (LOL) maps in Figure 1c and Figure 1d of super-B and graphene with isovalue 0.4 a.u. have been conducted by Multiwfn. ${ }^{22,23}$ All the $\pi$ bonds are well depicted in LOL picture. It is evident that the $\pi$ electrons from $p$ orbitals of $s p$ boron delocalized on the whole plane of super-B. All these delocalized large $\pi$ bonds, analogous with graphene, significantly contribute to the stability of super-B.Interestingly, the chemical bonds of super-B with $\pi(5 \mathrm{c}-3 \mathrm{e})$ bond have not been reported before in $2 \mathrm{D}$ materials and are quite different from the conventional bond in borophene allotrope, like three-centered bond in triangular lattice with/without vacancies. ${ }^{8,24}$

In addition, the similarity of delocalized large $\pi$ bonds between graphene and super-B is also reflected by the electronic configurations shown in Figure 2. The relationship between benzene $\left(\mathrm{C}_{6} \mathrm{H}_{6}\right)$ and graphene is the same as hydrogenated super- $\mathrm{B}\left(\mathrm{B}_{12} \mathrm{H}_{6}\right)$ and super- $\mathrm{B}$. The electronic structure of benzene is well-known as three completely delocalized $\pi$ orbitals, 6c2e $\pi$ bonds, ${ }^{25}$ shown in Figure 2b-2d based on the adaptive nature density partitioning (AdNDP). ${ }^{21} \mathrm{~B}_{12} \mathrm{H}_{6}$ is composed of three completely delocalized $12 \mathrm{c}-2 \mathrm{e} \pi$ bonds with a little electron-deficiency characteristic. Here, the completely delocalized $\pi$ bond in super-B is verified again. Besides, we also find that there is no 
(a)

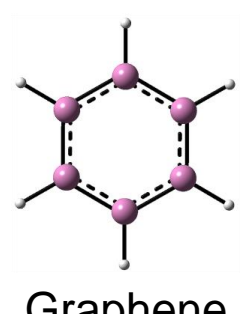

Graphene

(e)

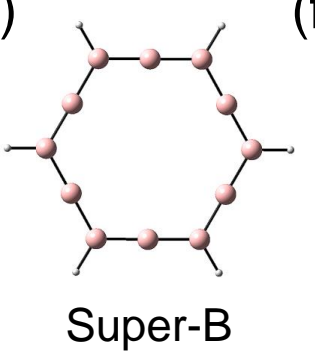

(b)

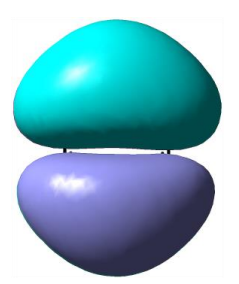

(c)

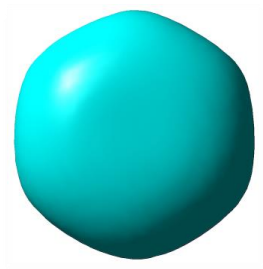

$36 \mathrm{c}-2 \mathrm{e} \pi$ bond

(f)

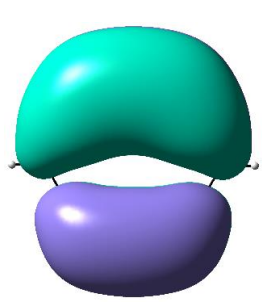

g)

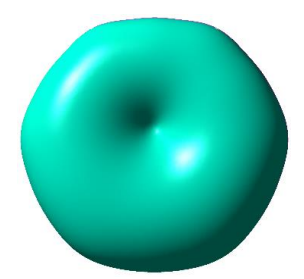

(d)

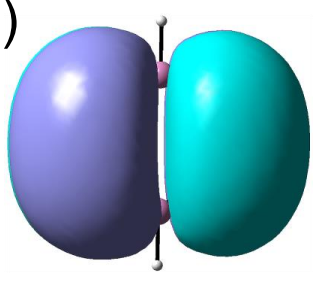

(h)

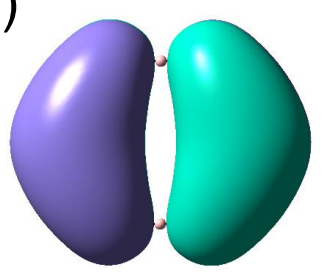

3 12c-2e $\pi$ bond

Figure 2: (a) (e) Optimized structures of benzene $\left(\mathrm{C}_{6} \mathrm{H}_{6}\right)$ and hydrogenated super- $\mathrm{B}\left(\mathrm{B}_{12} \mathrm{H}_{6}\right)$. Molecular orbitals of (b)(c)(d) 6c-2e $\pi$ bonds of benzene and (f)(g)(h) 12c-2e $\pi$ bonds of hydrogenated super-B based on the AdNDP method ${ }^{21}$ (white ball stands for hydrogen).

Jahn-Teller distortion in super-B by optimizing the atomic positions in a large supercell.

The cohesive energy $E_{c}$ is the amount of energy to break a material into isolated atoms. Calculated $E_{c}$ of super-B is $5.55 \mathrm{eV} /$ atom, only $0.1 \mathrm{eV} /$ atom higher than $\delta_{6}-\mathrm{B},{ }^{12}$ but more stable than the $\delta_{4}-\mathrm{B}^{26}$ shown in Table 2. For any mechanically stable $2 \mathrm{D}$ materials, a necessary, but not a sufficient condition must be satisfied: $\mathrm{C}_{11} \mathrm{C}_{22}-\mathrm{C}_{12}^{2}>0$ and $\mathrm{C}_{66}>0 .{ }^{27}$ The calculated elements of super-B are $\mathrm{C}_{11}=\mathrm{C}_{22}=146.0 \mathrm{GPa}$, $\mathrm{C}_{12}=138.1 \mathrm{GPa}$, and $\mathrm{C}_{66}=3.9 \mathrm{GPa}$, assuming an effective thickness of $0.384 \mathrm{~nm}$ (two van der Waals radius), which verifies the mechanical stability of super-B. The phonon dispersion is shown in the Supporting Information. Obviously, there are two linear LA and TA acoustic phonon branches, and a parabolic ZA around $\Gamma$-point. All frequencies are free from imaginary, confirming the lattice dynamical stability. Besides, we also implemented ab initio molecular dynamics simulations using canonical ensemble and Nosé-Hoover thermostat at $600 \mathrm{~K}$ for 10 ps. The movie, shown in the Supporting Information, indicates that the robustness of our predicted super-B. Besides, we find that super-B is non-magnetic, even having an odd number of electrons based on the first-principle calculation. Therefore, our calculation confirms that super-B is chemically, thermodynamically, mechanically, and dynamically stable.

Intriguingly, we find a similar pattern, called $\alpha$-graphyne, has been explored in the carbon counterpart but is not dynamically stable. ${ }^{28}$ However, inserting two threefold-coordinated carbon atoms into a $2 \mathrm{D}$ hexagonal lattice makes $\beta$-graphyne stable. ${ }^{29} \mathrm{We}$ did a similar analysis and found that the phonon dispersion of $\beta$ borophyne has large imaginary frequency, reflecting the significant distinction of electron orbitals between carbon and boron. Considering $\beta$-graphyne substructures and derivatives that have been experimentally synthesized in largearea nano-films, ${ }^{30,31}$ we are confident that the same experimental technique can be extended to the super-B and boron nanostructures on the horizon.

\section{Electronic structure and doping effect}

The projected electronic band structure and density of states (DOS) of super-B are shown in Figure 3a. Like graphene, $p_{z}$ orbital entirely dominates the electronic behavior around the Fermi level. We find that the intrinsic super- 
Table 1: The natural bond orbital (NBO) analysis of atomic notation $\mathrm{B}_{3}$ and $\mathrm{B}_{4}$ in Figure 1a including the number of occupancy with spin-up, spin-down electrons of boron and total of them, as well as contributions of $s, p$ and $d$ atomic orbitals.

\begin{tabular}{|c|c|c|c|c|c|c|c|c|}
\hline \multirow{2}{*}{ Center atoms } & \multirow{2}{*}{ Bonding atoms } & \multicolumn{3}{|c|}{ Occupancy } & \multicolumn{4}{|c|}{ Hybridization of center boron ${ }^{a}$} \\
\hline & & Spin-up & Spin-down & total & $s p^{\lambda}$ & $s(\%)$ & $p(\%)$ & $d(\%)$ \\
\hline $\mathrm{B}_{3}$ & $\mathrm{~B}_{1}$ & 0.95 & 0.95 & 1.90 & $s p^{1.99}$ & 33.37 & 66.53 & 0.10 \\
\hline $\mathrm{B}_{3}$ & $\mathrm{~B}_{2}$ & 0.95 & 0.95 & 1.90 & $s p^{1.99}$ & 33.37 & 66.53 & 0.10 \\
\hline $\mathrm{B}_{3}$ & $\mathrm{~B}_{4}$ & 0.95 & 0.95 & 1.90 & $s p^{2.02}$ & 33.06 & 66.84 & 0.10 \\
\hline $\mathrm{B}_{4}$ & $\mathrm{~B}_{3}$ & 0.95 & 0.95 & 1.90 & $s p^{1.00}$ & 49.99 & 50.00 & 0.01 \\
\hline $\mathrm{B}_{4}$ & $\mathrm{~B}_{5}$ & 0.95 & 0.95 & 1.90 & $s p^{1.00}$ & 49.99 & 50.00 & 0.01 \\
\hline
\end{tabular}

${ }^{a}$ The contribution of $s, p$ and $d$ orbitals of center boron is the average of spin-up, spin-down electrons of boron, and $s p^{\lambda}$ of center boron is the almost same for both spin-up and spin-down.

Table 2: The calculated lattice constants, planer or buckling, coordination number, symmetry and cohesive energy for $2 \mathrm{D}$ borophene allotropes $\chi_{3}, \beta_{12}, \delta_{4}, \delta_{6}$ and our proposed super-B.

\begin{tabular}{lllllll}
\hline \hline Structures & $a_{1}(\AA)$ & $a_{2}(\AA)$ & Planer & $Z$ & Symmetry & $E_{c}(\mathrm{eV} /$ atom $)$ \\
\hline$\chi_{3}-\mathrm{B}^{a}$ & 4.45 & 4.45 & Yes & 4,5 & $C \mathrm{mmm}$ & 5.723 \\
$\beta_{12}-\mathrm{B}^{a}$ & 2.92 & 5.07 & Yes & $4,5,6$ & $P \mathrm{mmm}$ & 5.712 \\
$\delta_{4}-\mathrm{B}^{a}$ & 2.93 & 3.28 & Yes & 4 & $P \mathrm{mmm}$ & 5.384 \\
$\delta_{6}-\mathrm{B}^{a}$ & 3.22 & 3.29 & No & 6 & $P \mathrm{mmn}$ & 5.662 \\
Super-B $^{b}$ & 5.45 & 5.45 & Yes & 2,3 & $P 6 / \mathrm{mmm}$ & 5.550 \\
\hline \hline
\end{tabular}

${ }^{a}$ Ref. ${ }^{12,26}{ }^{b}$ Present work. All results are based on the PBE functionals. 
(a)

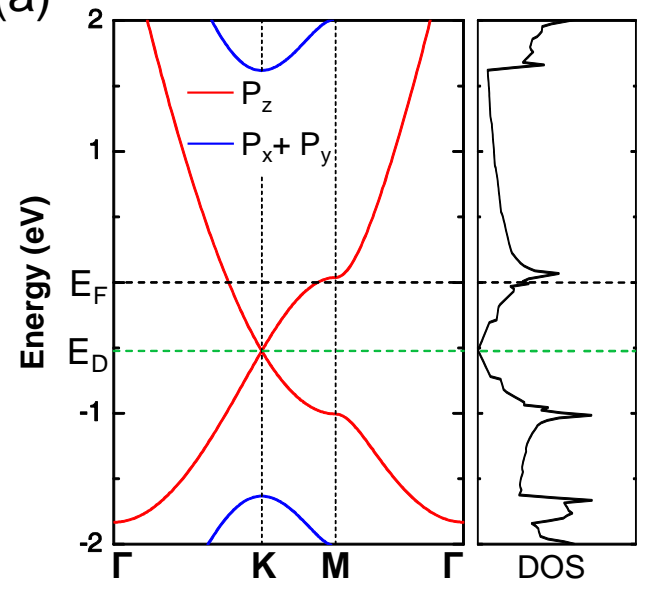

(b)

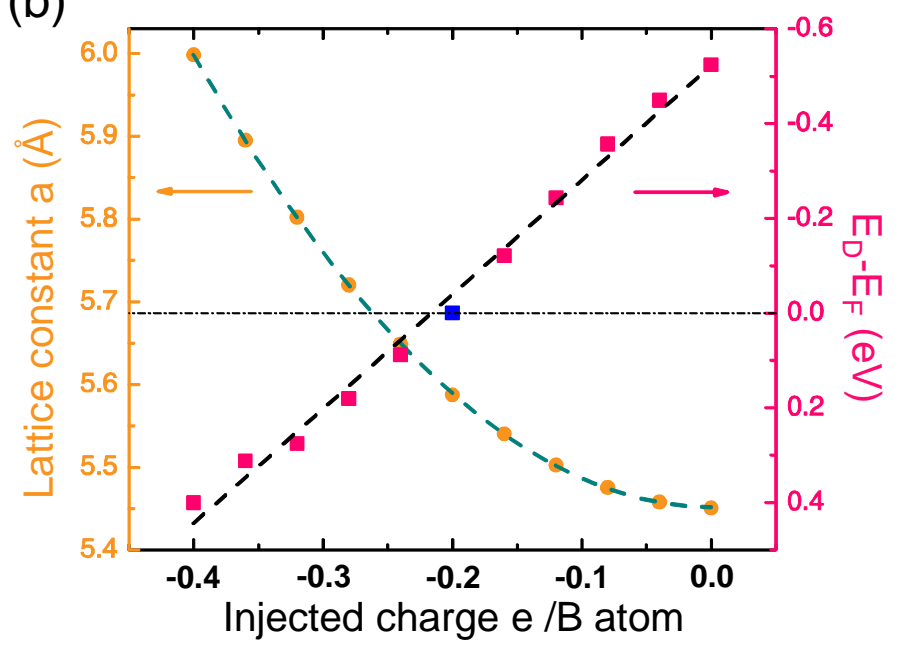

Figure 3: (a) Orbitally resolved band structure and total density of states for pristine superborophene. (b) The lattice constant and the position of Dirac cone $\left(E_{D}\right)$ with respect to the Fermi level $\left(E_{F}\right)$ as a function of injected charge. The green dashed line is a parabolic fitting of lattice constant with $0.999 \mathrm{R}$-Squared and the black dashed line is a linear fitting of the relative gap between $E_{F}$ and $E_{D}$ with 0.992 R-Squared. Blue square denotes the situation of $E_{F}=E_{D}$. The dashed horizontal lines are guided for your eyes.

$\mathrm{B}$ is metallic, which is analogous with $\alpha$-B, ${ }^{24}$ $\beta_{12}-\mathrm{B},{ }^{12}$ and other monolayer boron sheets. ${ }^{1,8}$ Below the $E_{F}$ around $0.52 \mathrm{eV}$, two $p_{z}$ bands cross each other, forming a standard Dirac cone $\left(E_{D}\right)$, which is verified by the total DOS in the right panel.

Recently, honeycomb borophene by extra doping of $1 \mathrm{e} / \mathrm{B}$ atom, identical with graphene's configuration, has been realized experimentally ${ }^{32}$ and theoretically. ${ }^{33}$ Here, we would like to explore how large doping could make super$\mathrm{B}$ a semimetal, like graphene. Since $E_{F}$ is larger than $E_{D}$ in the original state, one should provide a degree of hole doping to the super-B, shifting up the bands. The results are shown in Figure 3b. In the calculation, when adding or removing an amount of electrons (holes), the system will be compensated by the same amount uniform background charge of holes (electrons), retaining a continuous neutral condition. This standard method has been verified by many previous works. ${ }^{33}$

In each doping concentration, we optimized both lattice constants and atomic positions. On the one hand, the lattice constant of super-B, interestingly, can be well fitted by a quadratic polynomial with 0.999 R-Squared as a function of hole doping. Due to the special $s p, s p^{2}$ and $\pi(5 \mathrm{c}-3 \mathrm{e})$ bond, the bond length increases from $1.573 \AA$ to $1.613 \AA$ when injecting $-0.2 / \mathrm{B}$ and further reaches to $1.731 \AA$ at $-0.4 / \mathrm{B}$. This phenomenon is different from the previous orthogonal $\epsilon$-B allotrope ${ }^{33}$ in which doping has an anisotropic effect on the lattice constants. Due to the $P 6 / \mathrm{mmm}$ symmetry of super-B, the net charge has the same effect on both $\mathrm{a}_{1}$ and $\mathrm{a}_{2}$ lengths. In this sense, doping is an effective way to change the lattice constant of super-B and other 2D borophenes. ${ }^{33,34}$

On the other hand, doping will change the electronic band structure, especially the position of $E_{F}$. The relative position of $E_{D}$ with respect to $E_{F}$, in Figure $3 \mathrm{~b}$, decreases when increasing the injected holes. We find that the $E_{D^{-}} E_{F}$ can be well fitted by a linear function with 0.992 R-Squared, indicating a rigid band shift in super-B. - - 0.4/B doping corresponds to $1.44 \times 10^{14} \mathrm{~cm}^{-2}$, which is an accessible and reachable value in the current experimental technique, such as electrical gating and ionic liquid injection. Even though the rigid band shift is common in 2D transition metal dichalcogenides, ${ }^{35}$ we have not found a similar behavior in $2 \mathrm{D}$ boron allotrope heretofore. The reason 
(a)

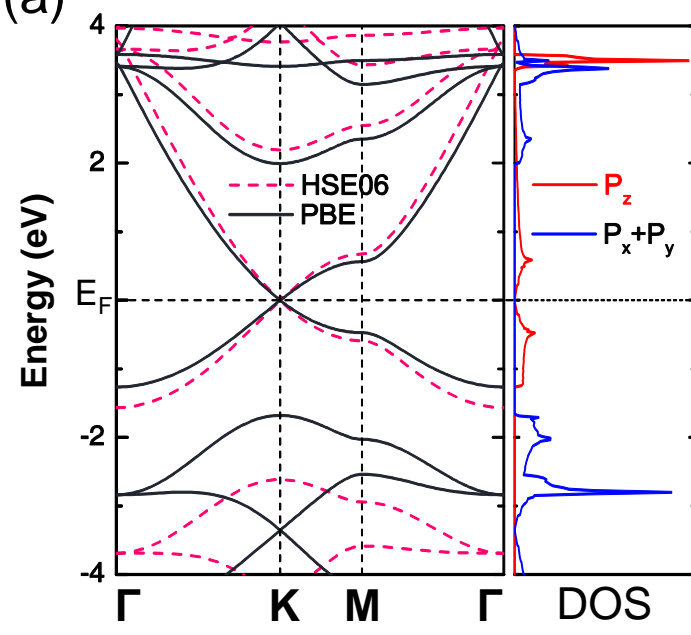

(c)

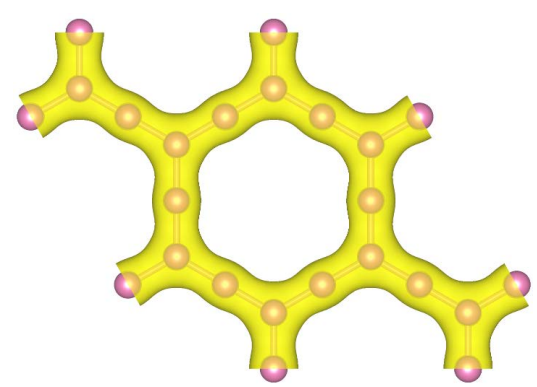

(b)

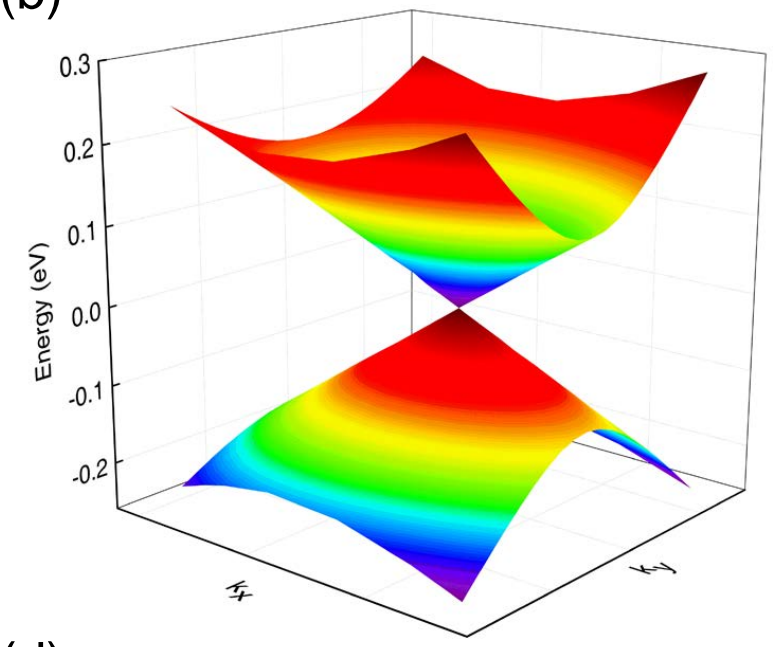

(d)

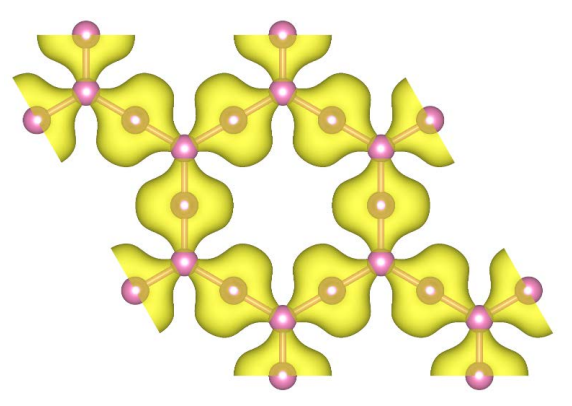

Figure 4: (a) Electronic band structures from PBE (black solid line) and HSE06 (magenta dashed line) methods and projected density of states (PBE level) of super-B with injected electrons $\langle\Delta Q\rangle$ $=-0.2 \mathrm{e} / \mathrm{B}$, corresponding to a doping level of $7.7 \times 10^{13} \mathrm{~cm}^{-2}$ that has been realized by electrical gatingand ionic liquid injection.(b) 3D Dirac cone formed by the valence and conduction bands in the vicinity of Dirac point. In the first Brillouin zone, the high symmetry $k$ points are: $\Gamma\left(\begin{array}{lll}0 & 0 & 0\end{array}\right)$, $\mathrm{K}(-1 / 32 / 30)$, and $\mathrm{M}(00.50)$. (c,d) The isosurfaces of partial charge densities for the (c) VBM and $(\mathrm{d}) \mathrm{CBM}$ of super-B with $\langle\Delta Q\rangle=-0.2 \mathrm{e} / \mathrm{B}$. The isosurface level value is $0.03 \mathrm{e} \AA^{-3}$.

behind it is that many $2 \mathrm{D}$ borophenes mainly possess three-centered bond characteristic, ${ }^{8,24}$ rather than $s p, s p^{2}$, and delocalized $\pi(5 \mathrm{c}-3 \mathrm{e})$ bond in super-B.

In order to further confirm the Dirac cone of super-B at $-0.2 / \mathrm{B}\left(7.7 \times 10^{13} \mathrm{~cm}^{-2}\right)$ doping concentration, we plot the electronic band and corresponding DOS in Figure 4a using PBE (black solid line) and standard HSE06 methods (pink dashed line). It shows that $p_{x}+p_{y}$ shift up or down using HSE06. However, the bands from $p_{z}$ orbital do not change its position, only having slightly different slopes of two linear bands when hybrid functional is applied. 3D band in the reciprocal space, in Figure $4 \mathrm{~b}$, further proves the band crossing at the K-point. Besides, we compare the band structures of $\alpha$-graphyne and hole-doped super-B in the Supporting Information. They look similar but they have different Fermi velocities $v_{F}$. Even though $v_{F}$ of super-B is smaller than $\alpha$ graphyne, we show the possibility to transform from metal to semimetal in 2D boron system by extra doping. Furthermore, we calculate the partial charge densities of valence band maximum (VBM) and conduction band minimum (CBM) of super-B in Figure $4 \mathrm{c}$ and $4 \mathrm{~d}$. VBM stems from the delocalized orbitals, resulting in large $\pi(5 \mathrm{c}-3 \mathrm{e})$ bond that has mentioned in Figure 1c, whereas CBM shows localized in-plane orbitals constituting the standard $s p$ and $s p^{2}$ bonds between boron atoms. This picture is well consistent with the chemical NBO analysis shown in Figure 1. 
(a)

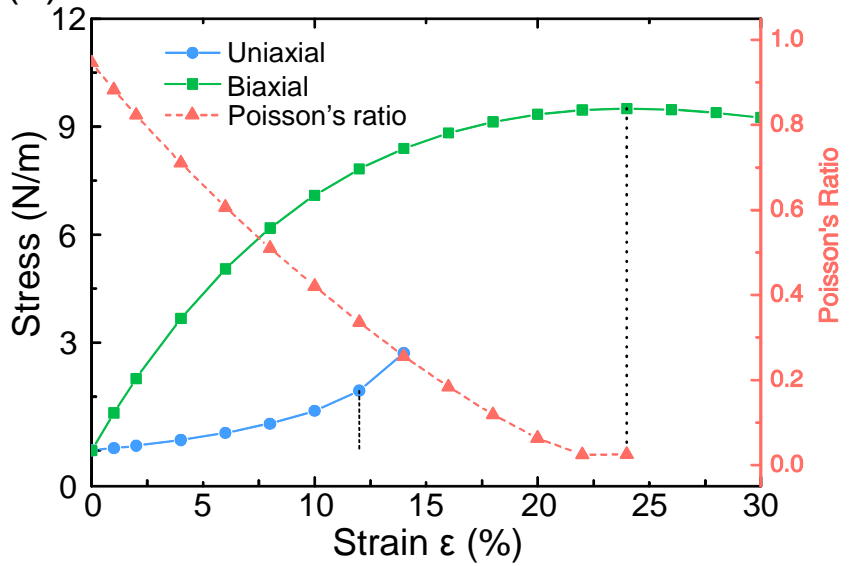

(b)

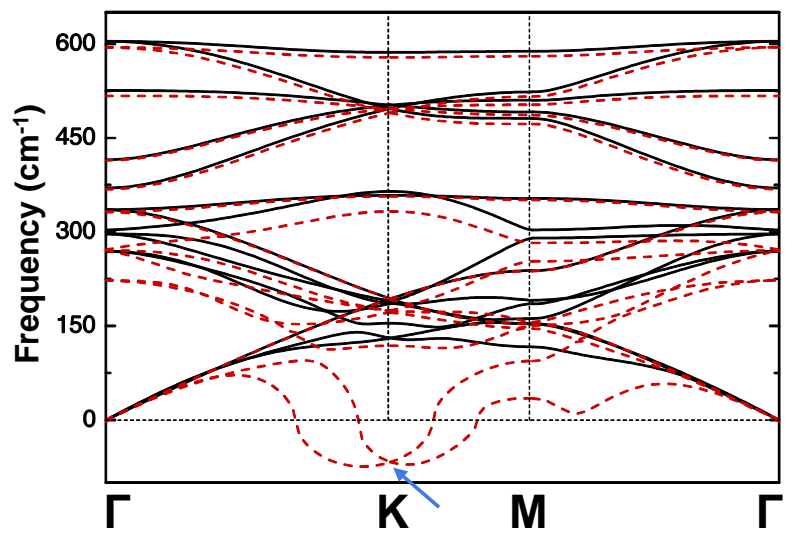

Figure 5: (a) Strain-stress curves of super-borophene while loaded along biaxial (green) and uniaxial (blue) directions, and Poisson's ratio (pink) under biaxial strain. (b) phonon dispersion under 24\% (solid black line) and 25\% (red dashed line) biaxial strain. The critical points of both types of strain are marked by vertical dashed lines in (a). The blue arrows illustrates the "Kohn anomaly" related to lattice instability.

\section{Mechanical property}

In practical applications, a large ideal strength of material is highly desired in flexible electronic devices. ${ }^{36-38}$ According to the definition, biaxial tensile strain can be expressed as $\varepsilon=a / a_{0}-1$, in which $a$ and $a_{0}$ are the stretched and pristine lattice constant of materials. As biaxial strain $\varepsilon$ increases, in Figure $5 \mathrm{a}$, the stress $\sigma$ in green line first linearly increases, then gradually saturates with a maximum value called ideal strength. ${ }^{27}$ The ideal strength of super-B is $9.50 \mathrm{~N} / \mathrm{m}$, which is the same order of many other borophenes. ${ }^{36,39}$ In each strain, we calculate the phonon dispersion to verify its stability. Smaller than $\varepsilon \leq 24 \%$, all phonon frequencies are positive, shown in Figure 5b (black line). When $\varepsilon=25 \%$, the system becomes unstable, which is a clear hint of the "Kohn anomaly" when reaching the ideal strength in super-B. $\varepsilon$ $=25 \%$ strain is also called breaking point which means a material physically breaks at its breaking point. This phenomenon has been studied in graphene extensively. ${ }^{34,40,41}$

For the uniaxial strain, the isotropically stress response of super-B is shown in Figure $5 \mathrm{a}$ in blue line. The ideal strength is $1.66 \mathrm{~N} / \mathrm{m}$ at $\varepsilon=12 \%$, relatively smaller than the biaxial strain (phonon dispersion is shown in the Supporting Information). Young's modulus $E$ is

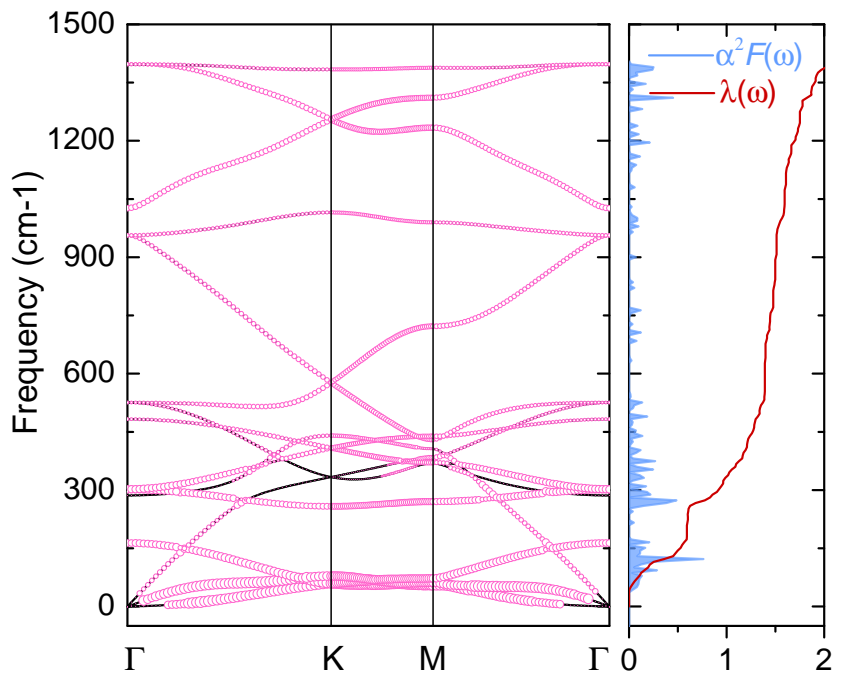

Figure 6: Phonon band structure and electron-phonon coupling (EPC) of the superborophene. The area of the pink circle is proportional to the EPC strength. The right panel is Eliashberg spectral function $\alpha^{2} F(\omega)$ and the total EPC constant $\lambda(\omega)$.

the slope of the strain-stress curve. A large $E$ means a rigid material. The calculated $E$ by linear fitting equals $5.85 \mathrm{~N} / \mathrm{m}$, indicating a soft mechanical property of super-B. We also verify this value by using elastic tensor formula ${ }^{27,42,43}$ $E=\left(\mathrm{C}_{11} \mathrm{C}_{22}-\mathrm{C}_{12} \mathrm{C}_{21}\right) / \mathrm{C}_{22}$. This ultrasoft elastic property of super-B may have great potential for designing flexible electronic devices. ${ }^{36,39}$ 
Table 3: Single-element superconductivity of graphene, silicene, phosphorene, stanene, $\chi_{3^{-}}, \beta_{12^{-}}$ , $\delta_{6^{-}}$and super-borophene.

\begin{tabular}{lccc}
\hline \hline Materials & $\lambda$ & $\omega_{\log }\left(\mathrm{cm}^{-1}\right)$ & $T_{c}^{\mu^{*}=0.1}(\mathrm{~K})$ \\
\hline Graphene $^{a}$ & 0.61 & 277.8 & 8.1 \\
Silicene $^{b}$ & 0.44 & 236.9 & 1.7 \\
Phosphorene $^{c}$ & 0.54 & 176.3 & 4.2 \\
Stanene $^{d}$ & 0.65 & 42.3 & 1.3 \\
$\chi_{3}-\mathrm{B}^{e}$ & 0.62 & 455.4 & 11.5 \\
$\beta_{12}-\mathrm{B}^{e}$ & 0.78 & 362.0 & 16.1 \\
$\delta_{6}-\mathrm{B}^{e}$ & 1.05 & 272.5 & 20.5 \\
Super-B $^{f}$ & 1.95 & 102.1 & 20.8 \\
\hline \hline
\end{tabular}

${ }^{\mathrm{a}} \mathrm{Li}$ doped graphene. ${ }^{44}{ }^{b}$ Silicene under $0.44 \mathrm{e} /$ atom doping. ${ }^{45}{ }^{c}$ Phosphorene under 0.10 e/atom doping. ${ }^{46}{ }^{d} \mathrm{Li}$ doped stanene. ${ }^{47}{ }^{e}$ Three experimental 2D borophenes. ${ }^{48}{ }^{f}$ Present work.

The Poisson's ratio $\nu$, defined as the negative sign ratio of lateral to applied strain, ${ }^{27,49}$ is shown in Figure 5a in a pink curve. The equilibrium super-B has a very large $\nu$ of 0.95 , more than 5 times of borophene with vacancy $=1 / 8^{36}$ and also 5 times of graphene. ${ }^{50}$ This ultrahigh of $\nu$ suggests that the response deformation of super-B is almost with the same amplitude when applied strain to the pristine structure. As strain increases, $\nu(\varepsilon)$ decreases significantly, indicating a gradual weakness of Poisson effect at large strain. The minimum $\nu(\varepsilon)$ is 0.03 at $\varepsilon=24 \%$, reducing to a final saturation near the critical point. ${ }^{49,50}$

\section{Superconductivity}

As an accepted rule of thumb, a good phononmediated superconductor may satisfy some of conditions: (i) a small average atomic mass $\bar{M}$, (ii) a large electronic density of states (DOS) at $E_{F}$, (iii) a large Debye temperature $\theta_{D}$. As $\theta_{D}$ is inversely proportional to $\bar{M}$, a small $\bar{M}$ generally means a large $\theta_{D}$. In this sense, hydrogen is a good candidate for superconductor. However, hydrogen is an insulator at normal condi- tion, having a very small DOS at $E_{F}$. Metallic boron, also with very small $\bar{M}$, is a promising candidate to satisfy all above criterion simultaneously. The famous bulk $\mathrm{MgB}_{2}$ in which magnesium is inserted into hexagonal boron, has an unprecedented high $T_{c}$ of $39 \mathrm{~K} .{ }^{6}$

According to the BardeenCooperSchrieffer (BCS) theory and Eliashberg equations, the spectral function can be expressed as ${ }^{51}$

$$
\alpha^{2} F(\omega)=\frac{1}{2 \pi N\left(E_{F}\right)} \sum_{\mathbf{q} \nu} \delta\left(\omega-\omega_{\mathbf{q} \nu}\right) \frac{\gamma_{\mathbf{q} \nu}}{\hbar \omega_{\mathbf{q} \nu}},
$$

where $N, \omega_{\mathbf{q} \nu}$, and $\gamma_{\mathbf{q} \nu}$ are the electronic density of states at $E_{F}$, phonon frequency and linewidth for phonon modes $\lambda$ with the wave vector $\mathbf{q}$. The total EPC constant can be obtained when making a summation of spectral function over the first Brillouin zone

$$
\lambda=\sum_{\mathbf{q} \nu} \lambda_{\mathbf{q} \nu}=2 \int \frac{\alpha^{2} F(\omega)}{\omega} d \omega,
$$

Finally, based on the AllenDynes formula, ${ }^{52}$ the critical temperature $T_{c}$ of superconductor can be estimated by

$$
T_{c}=\frac{\omega_{\log }}{1.2} \exp \left[\frac{-1.04(1+\lambda)}{\lambda\left(1-0.62 \mu^{*}\right)-\mu^{*}}\right],
$$

in which $\mu^{*}$ is the effective screened Coulomb repulsion constant (generally $0.10 \sim 0.15$ ) and $\omega_{\log }$ reads

$$
\omega_{\log }=\exp \left[\frac{2}{\lambda} \int \frac{d \omega}{\omega} \alpha^{2} F(\omega) \log (\omega)\right] .
$$

The calculated phonon dispersion, EPC, and spectral function of super-B are shown in Figure 6. The area of pink circle is proportional to the strength of EPC. The largest EPC strength stems from the ZA, TA and one special optical phonon mode. For the purely flat $2 \mathrm{D}$ materials, such as graphene, ZA mode must satisfy a symmetry-based selection rule. ${ }^{53}$ Therefore, for 2D materials, ZA modes generally dominant the heat transport and show a giant anharmonicity (large Grüneisen parameter).

As the vibrational direction of this optical phonon is parallel to the $z$ axis, we call it $O_{z}$ 
(a)

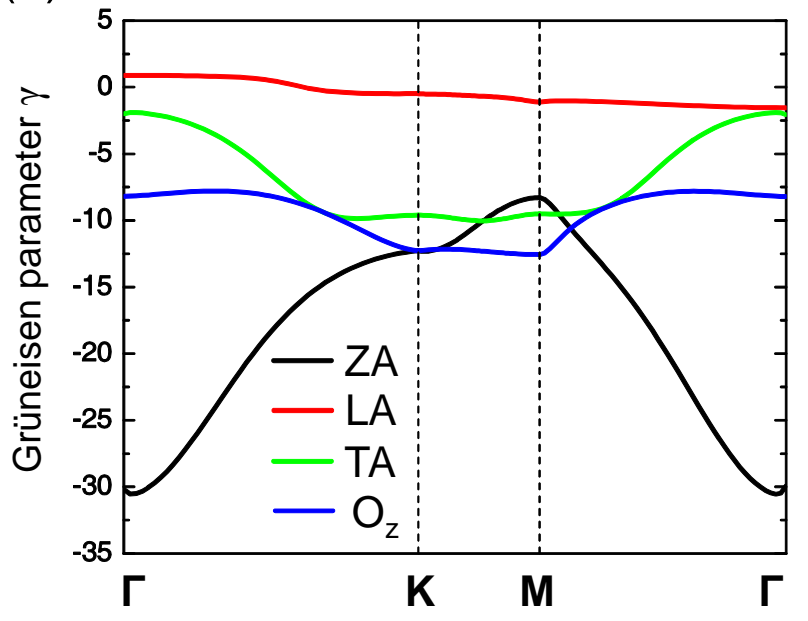

(b)

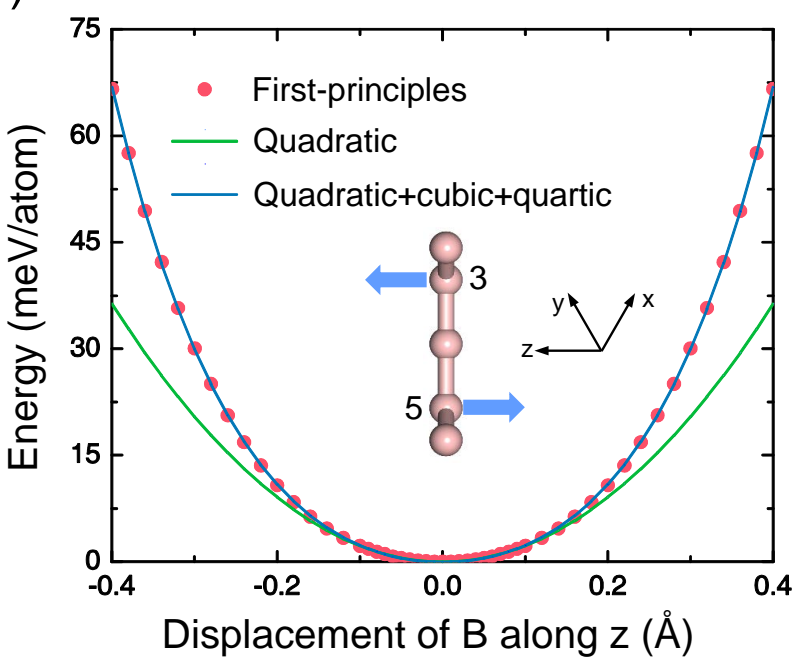

Figure 7: (a) Grüneisen parameter $\gamma$ of three acoustic and one optical phonon branches in the first Brillouin zone. (b) Total energy as a function of displacement of $O_{z}$ phonon mode, in which $\mathrm{B}_{1}$, $\mathrm{B}_{2}$, and $\mathrm{B}_{4}$, shown in Figure 1a, are static but $\mathrm{B}_{3}$ and $\mathrm{B}_{5}$ make movements in an opposite direction as an inset.

mode for the sake of simplicity. The $O_{z}$ mode, anomalously, drops into the acoustic phonon regions which is in spirit similar with recent discovered $2 \mathrm{D}$ tellurene. ${ }^{42}$ The low optical $O_{z}$ mode makes it easier to satisfy the energy and momentum conservation simultaneously, leading to a large phase space of phonon-phonon scattering. ${ }^{42}$ The EPC strength of this $O_{z}$ mode firstly increases from $\Gamma$ to $\mathrm{K}$, and $\mathrm{M}$ and then decreases when approaching $\Gamma$ again.

The corresponding Eliashberg spectral function $\alpha^{2} F(\omega)$, in Figure 6, also reveals that this $O_{z}$ mode enhances the EPC in the lowfrequency of acoustic phonon region. The total EPC constant $\lambda$ and critical temperature $T_{c}$, according to the Eq. (1)-(4), are shown in Table 3 . The phonon-mediated $T_{c}$ of super-B is $20.8 \mathrm{~K}$ with $\lambda=1.95$ (at $\mu=0.1$ ). We also list some other related $2 \mathrm{D}$ materials and borophene allotropes. Our super-B has the first-class intrinsically critical temperature $T_{c}$ of $20.8 \mathrm{~K}$, which is comparable and even a little higher than the $\delta_{6}-\mathrm{B}(20.5 \mathrm{~K})$. To the best of knowledge, super-B has the highest intrinsically critical temperature $T_{c}$ in single-element superconductors at ambient condition.

Furthermore, Profeta et al. ${ }^{44}$ proposed a seminal strategy to significantly enhanced the $T_{c}$ of $\mathrm{LiC}_{6}$ by inserting the metal lithium due to a large density of state at Fermi level. We hope $T_{c}$ of super-B can also be further enhanced by a similar method, such as metal potassium. ${ }^{54}$

After analysis of separated phonon modes, we find that the high $T_{c}$ of super-B mainly stems from the giant phonon anharmonicity.ZA, TA and $O_{z}$ phonon modes are the three main contributions to the large EPC, and finally large $T_{c}$. The Grüneisen parameter $\gamma$, in Figure $7 \mathrm{a}$, shows volume response as a function of phonon thermal vibration in super-B. A large $\gamma$ means a strong anharmonicity in chemical bonds of materials. ${ }^{42,55,56}$ The largest value of $\gamma$ is around -30 , belonging to the ZA mode in black line. The secondary is $O_{z}$ branch in blue line having an average value of -10 . This unusually low optical phonon branch would enhance the phononphonon scattering, as well as electron-phonon scattering. Notably, this is also evidenced by the fact that the soft phonon modes are eliminated with applied strain in Figure 5b. Specifically, the strong acoustic-acoustic phonon and acoustic-optical phonon interactions lead to a large phonon anharmonicity. This giant anharmonicity, finally, brings about the large peak in the Eliashberg spectral function $\alpha^{2} F(\omega)$ in the right panel in Figure 6. Besides, based on the Eq. (2), we obtain that there is $60 \% \lambda$ below $300 \mathrm{~cm}^{-1}$ in the total EPC contributions, 
which verifies that low frequency significantly is responsible for this unusually large $\lambda$.

To further explore the phonon anharmonic property in super-B, we calculate the total energy as a function of displacement in phonon $O_{z}$ mode, in which $\mathrm{B}_{1}, \mathrm{~B}_{2}$, and $\mathrm{B}_{4}$ shown in Figure $1 \mathrm{a}$ are static but $\mathrm{B}_{3}$ and $\mathrm{B}_{5}$ make movements in an opposite direction, in Figure 7b. ZA and TA modes also have similar behaviors. The first principle results can be well fitted by a polynomial expression up to quartic $E(u)=225.56 u^{2}+1203.01 u^{4}$ in the blue line. However, harmonic approximation (green line) cannot produce a good agreement. The ratio between quartic and quadratic terms is $A_{4} / A_{2} \approx 5.33$, indicating a quite large anharmonicity in super-B, which finally leads to a strong interaction between electrons and phonons. To the best of knowledge, superconductor $\mathrm{MgB}_{2}$ binary alloy ${ }^{57}$ and $2 \mathrm{D}$ tellurene with high figure of merit $z T^{42,58}$ also exhibit very large phonon anharmonicity.

For the band structure, we consider the distorted and undistorted super-B due to the $O_{z}$ phonon mode. The result is shown in the Supporting Information. The band splitting between the undistorted and distorted structures in super-B is around $0.07 \mathrm{eV}$. Although this number is smaller than the $\operatorname{MgB}_{2}\left(T_{c}=39 \mathrm{~K}\right){ }^{6}$ its impact is significantly large. We mark a special area with a blue circle which is close to the Fermi level and more importantly, and its DOS is quite large due to the relatively flat band. Hence, giant anharmonicity of this $O_{z}$ phonon mode intensifies the DOS around the Fermi level, then stimulates the EPC and finally leads to a high $T_{c}$.

\section{Discussion}

Similar to carbon nanotube, boron can also be rolled into a cylinder. ${ }^{24,26}$ We optimize singledwalled borophene nanotubes (SWBNTs) with different diameters and edge shapes (armchair or zigzag). We find that all SWBNTs are quite stable remaining a good cylinder without any buckling or collapse. Results are shown in the table of the Supporting Information. The most important result is that SWBNTs are always good metal. In order to further confirm the metallic property, for example, we plot the DOS of zigzag $(8,0)$ and armchair $(8,8)$ tubes in the Supporting Information. At the $E_{F}$, DOS is significantly enhanced when rolling into a cylinder, which is irrelevant to the type of edge shapes. This probably will, in turn, further amplify the $T_{c}$ of SWBNTs according to the rule (ii) mentioned above. According to the previously seminal works, ${ }^{59} T_{c}$ will be unquestionably enhanced by rolling sheets into tubes as a result of opening new electron-phonon scattering channels. However, due to formidable computing requirement, currently, one is quite difficult to simulate the EPC in SWCNTs and SWBNTs based on the accurate first-principle calculations. As one of the nearest neighbors of carbon, super-B and SWBNTs are promising superconductors by using the similar method of carbon. ${ }^{44}$

Compared with $\chi_{3^{-}}, \beta_{12^{-}}$, and $\delta_{6}-\mathrm{B}$, super-B is a metastable phase of borophene. In retrospect, the most stable phase of $2 \mathrm{D}$ silicon is silicene with 18 atoms in the primitive cell, ${ }^{60}$ rather than the phase with 2 atoms. ${ }^{61}$ Even though the latter is much less stable than the previous, the latter, at present, could be easily grown with very accurate experimental technique. ${ }^{62,63}$ Similarly, blue phosphorus, ${ }^{64}$ as a metastable of black phosphorus, ${ }^{65}$ has also been successfully grown on $\mathrm{Au}(111) .{ }^{66,67}$ The cohesive energy $E_{c}$ of the experimentally attainable silicene with 2 atoms and phosphorene are $3.71 \mathrm{eV} /$ atom $^{62,63}$ and $3.61 \mathrm{eV} /$ atom, ${ }^{65,68}$ separately. A large $E_{c}$ indicates a strong chemical bond in materials. The $E_{c}$ of super-B is $5.55 \mathrm{eV}$, indicating a strong and robust chemical bond to maintain stability. Furthermore, due to the complex chemical environment and variable substrate effect, a recently discovered borophene has been synthesised. ${ }^{11}$ Notably, it is a novel metastable borophene, also less stable than the $\chi_{3^{-}}, \beta_{12^{-}}$, and $\delta_{6}-\mathrm{B}$ allotropes.

Very recently, a cutting-edge experimental technique, called reactive molecular beam epitaxy method, has been successfully used to attain the freestanding crystalline oxide perovskites. ${ }^{69}$ Borophene, like oxide perovskites, 
has no counterpart in bulk material. Hence, except for the metal substrates, this recently developed approach may shed light on the experimental realization of super-B and other boronrelated nanostructures.

Furthermore, a 2D atomic layer with hexagonal boron network has been bottom-up synthesized freshly. The main structure in their work $^{54}$ is exactly our predicted super-B, which further verifies the correctness of our theoretical calculation and the importance of our work.

As a matter of fact, for strong coupling systems $(\lambda>1.5)$, superconducting temperature $T_{c}$ should be calculated using a more general expression according to Allen and Dynes ${ }^{52}$

$$
\begin{gathered}
T_{c}=\frac{f_{1} f_{2} \omega_{\text {log }}}{1.2} \exp \left[-\frac{1.04(1+\lambda)}{\lambda-\mu^{*}(1+0.62 \lambda)}\right], \\
f_{1}=\left[1+\left(\lambda / \Lambda_{1}\right)^{3 / 2}\right]^{1 / 3}, \\
f_{2}=1+\frac{\left(\bar{\omega}_{2} / \omega_{l o g}-1\right) \lambda^{2}}{\lambda^{2}+\Lambda_{2}^{2}}
\end{gathered}
$$

where $f_{1}$ and $f_{2}$ represent the strong-coupling correction and shape correction, respectively. The parameters $\Lambda_{1}$ and $\Lambda_{2}$ are given by ${ }^{52}$

$$
\begin{gathered}
\Lambda_{1}=2.46\left(1+3.8 \mu^{*}\right), \\
\Lambda_{2}=1.82\left(1+6.3 \mu^{*}\right)\left(\bar{\omega}_{2} / \omega_{l o g}\right),
\end{gathered}
$$

in which the general moment is defined as ${ }^{52}$

$$
<\omega^{n}>=\frac{2}{\lambda} \int d \omega \alpha^{2} F(\omega) \omega^{n-1},
$$

According to Eq. (5)-(10), the calculated $T_{c}$ is $25.3 \mathrm{~K}$ when $\mu^{*}=0.1$. Note that this result is larger than $20.8 \mathrm{~K}$ based on Eq. (3), further confirming our conclusion that super$\mathrm{B}$ has the highest critical temperature $T_{c}$ in single-element superconductors at ambient conditions.

In summary, we have predicted a previously unknown monolayer borophene by first principles. It has good thermal, dynamical, and mechanical stability compared with many other typical borophenes. We have found that super$\mathrm{B}$ has a fascinating chemical bond environment consisting of standard $s p, s p^{2}$ hybridizations and delocalized five-center three-electrons $\pi(5 \mathrm{c}-$ 3e) bonds, based on the NBO analysis. This exceptional electronic structure plays a crucial role in stabilizing the super-B chemically. Meanwhile, By extra doping, super-B can be transformed into a Dirac material from pristine metal. Like graphene, it also has a superior flexibility. Furthermore, due to the small atomic mass and large density of state at Fermi level, super-B has the highest intrinsically critical temperature $T_{c}$ of $25.3 \mathrm{~K}$ in single-element superconductors at ambient condition. We have attributed this high $T_{c}$ of super-B to the giant anharmonicity of two linear acoustic phonon branches and an unusually low optic $O_{z}$ phonon mode.

\section{Author Information}

\section{Corresponding Author}

*E-mail: zhibin.gao@nus.edu.sg

\section{ORCID}

Zhibin Gao: 0000-0002-6843-381X

\section{Supporting Information}

The Supporting Information is available free of charge on the ACS Publications website via the Internet at https://pubs.acs.org/journal/aamick. Phonon dispersion of super-B, electronic band structures of pristine $\alpha$-graphyne and super-B under $-0.2 \mathrm{e} / \mathrm{B}$ doping, and movies of molecular dynamics simulations at $\mathrm{T}=600 \mathrm{~K}$ at the end of 10 ps.

\section{Notes}

The authors declare no competing financial interest.

\section{Acknowledgement}

We acknowledge David Tománek for many fruitful discussions and good suggestions.We also thank Hanyu Liu for valuable discussions and kind help. M.L. acknowledges useful discussions with Xiang Zhao. TheHPCplatformofXi'anJiaotongUniversityis highlyappreciated. This work is supported by an MOE tier 1 grant R-144-000-402-114. 


\section{References}

(1) Li, W.-L.; Chen, X.; Jian, T.; Chen, T.T.; Li, J.; Wang, L.-S. From Planar Boron Clusters to Borophenes and Metalloborophenes. Nature Rev. Chem. 2017, 1, 0071.

(2) Sergeeva, A. P.; Popov, I. A.; Piazza, Z. A.; Li, W.-L.; Romanescu, C.; Wang, L.-S.; Boldyrev, A. I. Understanding Boron Through Size-Selected Clusters: Structure, Chemical Bonding, and Fluxionality. Acc. Chem. Res. 2014, 47, 1349 1358.

(3) Wang, L.-S. Photoelectron Spectroscopy of Size-Selected Boron Clusters: From Planar Structures to Borophenes and Borospherenes. Int. Rev. Phys. Chem. 2016, 35, 69-142.

(4) Eberhardt, W.; Crawford Jr, B.; Lipscomb, W. N. The Valence Structure of the Boron Hydrides. J. Chem. Phys. 1954, 22, 989-1001.

(5) Lipscomb, W. N. The Boranes and Their Relatives. Science 1977, 196, 1047-1055.

(6) Nagamatsu, J.; Nakagawa, N.; Muranaka, T.; Zenitani, Y.; Akimitsu, J. Superconductivity at $39 \mathrm{~K}$ in Magnesium Diboride. Nature 2001, 410, 63.

(7) Feng, B.; Sugino, O.; Liu, R.-Y.; Zhang, J.; Yukawa, R.; Kawamura, M.; Iimori, T.; Kim, H.; Hasegawa, Y.; Li, H. Dirac Fermions in Borophene. Phys. Rev. Lett. 2017, 118, 096401.

(8) Penev, E. S.; Bhowmick, S.; Sadrzadeh, A.; Yakobson, B. I. Polymorphism of Two-Dimensional Boron. Nano Lett. 2012, 12, 2441-2445.

(9) Liu, Y.; Penev, E. S.; Yakobson, B. I. Probing The Synthesis of TwoDimensional Boron by First-Principles Computations. Angew. Chem. Int. Ed. 2013, 52, 3156-3159.
(10) Zhang, Z.; Yang, Y.; Gao, G.; Yakobson, B. I. Two-Dimensional Boron Monolayers Mediated by Metal Substrates. Angew. Chem. Int. Ed. 2015, 54, 1302213026 .

(11) Mannix, A. J.; Zhou, X.-F.; Kiraly, B.; Wood, J. D.; Alducin, D.; Myers, B. D.; Liu, X.; Fisher, B. L.; Santiago, U.; Guest, J. R. Synthesis of Borophenes: Anisotropic, Two-Dimensional Boron Polymorphs. Science 2015, 350, 15131516.

(12) Feng, B.; Zhang, J.; Zhong, Q.; Li, W.; Li, S.; Li, H.; Cheng, P.; Meng, S.; Chen, L.; Wu, K. Experimental Realization of Two-Dimensional Boron Sheets. Nat. Chem. 2016, 8, 563.

(13) Kresse, G.; Furthmüller, J. Efficient Iterative Schemes for $a b$ initio Total-Energy Calculations Using A Plane-Wave Basis Set. Phys. Rev. B: Condens. Matter Mater. Phys. 1996, 54, 11169-11186.

(14) Kresse, G.; Furthmüller, J. Efficiency of ab-initio Total Energy Calculations for Metals and Semiconductors Using A Plane-Wave Basis Set. Comput. Mater. Sci. 1996, 6, 15-50.

(15) Kresse, G.; Hafner, J. Ab initio MolecularDynamics Simulation of The LiquidMetal-Amorphous-Semiconductor Transition in Germanium. Phys. Rev. B: Condens. Matter Mater. Phys. 1994, 49, 14251-14269.

(16) Perdew, J. P.; Burke, K.; Ernzerhof, M. Generalized Gradient Approximation Made Simple. Phys. Rev. Lett. 1996, rr., 3865-3868.

(17) Heyd, J.; Scuseria, G. E.; Ernzerhof, M. Hybrid Functionals Based on a Screened Coulomb Potential. J. Chem. Phys. 2003, 118, 8207-8215.

(18) Krukau, A. V.; Vydrov, O. A.; Izmaylov, A. F.; Scuseria, G. E. Influence of The Exchange Screening Parameter 
on The Performance of Screened Hybrid Functionals. J. Chem. Phys. 2006, 125, 224106 .

(19) Giannozzi, P.; Baroni, S.; Bonini, N.; Calandra, M.; Car, R.; Cavazzoni, C.; Ceresoli, D.; Chiarotti, G. L.; Cococcioni, M.; Dabo, I. QUANTUM ESPRESSO: A Modular and Open-Source Software Project for Quantum Simulations of Materials. J. Phys.: Condens. Matter 2009, 21, 395502.

(20) Frisch, M.; Trucks, G.; Schlegel, H.; Scuseria, G.; Robb, M.; Cheeseman, J.; Scalmani, G.; Barone, V.; Petersson, G.; Nakatsuji, H. Gaussian 16. Gaussian 16, revision A.03; Gaussian, Inc.: Wallingford, CT, 2016.

(21) Zubarev, D. Y.; Boldyrev, A. I. Developing Paradigms of Chemical Bonding: Adaptive Natural Density Partitioning. Phys. Chem. Chem. Phys. 2008, 10, 5207-5217.

(22) Schmider, H.; Becke, A. Chemical Content of The Kinetic Energy Density. J. Mol. Struct.: THEOCHEM 2000, 527, 51-61.

(23) Lu, T.; Chen, F. Multiwfn: A Multifunctional Wavefunction Analyzer. J. Comput. Chem. 2012, 33, 580-592.

(24) Tang, H.; Ismail-Beigi, S. Novel Precursors for Boron Nanotubes: The Competition of Two-Center and Three-Center Bonding in Boron Sheets. Phys. Rev. Lett. 2007, 99, 115501.

(25) Popov, I. A.; Boldyrev, A. I. Chemical Bonding In Coronene, Isocoronene, and Circumcoronene. Eur. J. Org. Chem. 2012, 2012, 3485-3491.

(26) Wu, X.; Dai, J.; Zhao, Y.; Zhuo, Z.; Yang, J.; Zeng, X. C. Two-Dimensional Boron Monolayer Sheets. ACS Nano 2012, 6, 7443-7453.
(27) Gao, Z.; Dong, X.; Li, N.; Ren, J. Novel Two-Dimensional Silicon Dioxide with InPlane Negative Poissons Ratio. Nano Lett. 2017, 17, 772-777.

(28) Longuinhos, R.; Moujaes, E. A.; Alexandre, S. S.; Nunes, R. Theoretical Chemistry of $\alpha$-Graphyne: Functionalization, Symmetry Breaking, and Generation of Dirac-Fermion Mass. Chem. Mater. 2014, 26, 3701-3708.

(29) Malko, D.; Neiss, C.; Viñes, F.; Görling, A. Competition for Graphene: Graphynes with Direction-Dependent Dirac Cones. Phys. Rev. Lett. 2012, 108, 086804.

(30) Li, G.; Li, Y.; Liu, H.; Guo, Y.; Li, Y.; Zhu, D. Architecture of Graphdiyne Nanoscale Films. Chem. Commun. 2010, 46, 3256-3258.

(31) Inagaki, M.; Kang, F. Graphene Derivatives: Graphane, Fluorographene, Graphene Oxide, Graphyne and Graphdiyne. J. Mater. Chem. A 2014, 2, 13193-13206.

(32) Li, W.; Kong, L.; Chen, C.; Gou, J.; Sheng, S.; Zhang, W.; Li, H.; Chen, L.; Cheng, P.; Wu, K. Experimental Realization of Honeycomb Borophene. Sci. bull. 2018, 63, 282-286.

(33) Liu, D.; Tomnek, D. Effect of Net Charge on the Relative Stability of 2D Boron Allotropes. Nano Lett. 2019, 19, 1359-1365.

(34) Si, C.; Duan, W.; Liu, Z.; Liu, F. Electronic Strengthening of Graphene by Charge Doping. Phys. Rev. Lett. 2012, 109, 226802.

(35) Gao, Z.; Zhou, Z.; Tománek, D. Degenerately Doped Transition Metal Dichalcogenides as Ohmic Homojunction Contacts to Transition Metal Dichalcogenide Semiconductors. ACS Nano 2019, 13, 51035111 . 
(36) Zhang, Z.; Yang, Y.; Penev, E. S.; Yakobson, B. I. Elasticity, Flexibility, and Ideal Strength of Borophenes. Adv. Funct. Mater. 2017, 27, 1605059.

(37) Liu, A. Y.; Cohen, M. L. Prediction of New Low Compressibility Solids. Science 1989, 245, 841-842.

(38) Si, C.; Liu, Z.; Duan, W.; Liu, F. FirstPrinciples Calculations on the Effect of Doping and Biaxial Tensile Strain on Electron-Phonon Coupling in Graphene. Phys. Rev. Lett. 2013, 111, 196802.

(39) Wang, H.; Li, Q.; Gao, Y.; Miao, F.; Zhou, X.-F.; Wan, X. Strain Effects on Borophene: Ideal Strength, Negative Possions Ratio and Phonon Instability. New J. Phys. 2016, 18, 073016.

(40) Yan, J.; Henriksen, E. A.; Kim, P.; Pinczuk, A. Observation of Anomalous Phonon Softening in Bilayer Graphene. Phys. Rev. Lett. 2008, 101, 136804.

(41) Lazzeri, M.; Mauri, F. Nonadiabatic Kohn Anomaly in a Doped Graphene Monolayer. Phys. Rev. Lett. 2006, 97, 266407.

(42) Gao, Z.; Tao, F.; Ren, J. Unusually Low Thermal Conductivity of Atomically Thin 2D Tellurium. Nanoscale 2018, 10, 12997-13003.

(43) Wang, Y.; Gao, Z.; Zhou, J. Ultralow Lattice Thermal Conductivity and Electronic Properties of Monolayer 1T Phase Semimetal $\mathrm{SiTe}_{2}$ and $\mathrm{SnTe}_{2}$. Physica $E$ 2019, 108, 53-59.

(44) Profeta, G.; Calandra, M.; Mauri, F. Phonon-Mediated Superconductivity in Graphene by Lithium Deposition. Nat. Phys. 2012, 8, 131-134.

(45) Wan, W.; Ge, Y.; Yang, F.; Yao, Y. Phonon-Mediated Superconductivity in Silicene Predicted by First-Principles Density Functional Calculations. Europhys. Lett. 2013, 104, 36001.
(46) Shao, D.; Lu, W.; Lv, H.; Sun, Y. Electron-Doped Phosphorene: A Potential Monolayer Superconductor. Europhys. Lett. 2014, 108, 67004.

(47) Shaidu, Y.; Akin-Ojo, O. First Principles Predictions of Superconductivity in Doped Stanene. Comput. Mater. Sci. 2016, 118, 11-15.

(48) Penev, E. S.; Kutana, A.; Yakobson, B. I. Can Two-Dimensional Boron Superconduct? Nano Lett. 2016, 16, 2522-2526.

(49) Gao, Z.; Liu, D.; Tománek, D. TwoDimensional Mechanical Metamaterials with Unusual Poisson Ratio Behavior. Phys. Rev. Applied 2018, 10, 064039.

(50) Liu, F.; Ming, P.; Li, J. Ab initio Calculation of Ideal Strength and Phonon Instability of Graphene Under Tension. Phys. Rev. B: Condens. Matter Mater. Phys. 2007, 76, 064120.

(51) Bardeen, J.; Cooper, L. N.; Schrieffer, J. R. Microscopic Theory of Superconductivity. Phys. Rev. 1957, 106, 162-164.

(52) Allen, P. B.; Dynes, R. C. Transition Temperature of Strong-Coupled Superconductors Reanalyzed. Phys. Rev. B: Condens. Matter Mater. Phys. 1975, 12, 905-922.

(53) Lindsay, L.; Broido, D. A.; Mingo, N. Flexural Phonons and Thermal Transport in Graphene. Phys. Rev. B: Condens. Matter Mater. Phys. 2010, 82, 115427.

(54) Kambe, T.; Hosono, R.; Imaoka, S.; Yamamoto, K. Solution Phase Mass Synthesis of 2D Atomic Layer With Hexagonal Boron Network. J. Am. Chem. Soc. 2019, 141, 12984-12988.

(55) Gao, Z.; Li, N.; Li, B. Heat Conduction and Energy Diffusion In MomentumConserving One-Dimensional Full-Lattice Ding-A-Ling Model. Phys. Rev. E 2016, 93, 022102. 
(56) Gao, Z.; Li, N.; Li, B. Stretch Diffusion and Heat Conduction In One-dimensional Nonlinear Lattices. Phys. Rev. E 2016, 93, 032130 .

(57) Yildirim, T.; Gülseren, O.; Lynn, J. W.; Brown, C. M.; Udovic, T. J.; Huang, Q.; Rogado, N.; Regan, K. A.; Hayward, M. A.; Slusky, J. S.; He, T.; Haas, M. K.; Khalifah, P.; Inumaru, K.; Cava, R. J. Giant Anharmonicity and Nonlinear Electron-Phonon Coupling in $\mathrm{MgB}_{2}$ : A Combined First-Principles Calculation and Neutron Scattering Study. Phys. Rev. Lett. 2001, 87, 037001.

(58) Gao, Z.; Liu, G.; Ren, J. High Thermoelectric Performance in Two-Dimensional Tellurium: An ab initio Study. ACS Appl. Mater. Interfaces 2018, 10, 40702-40709.

(59) Benedict, L. X.; Crespi, V. H.; Louie, S. G.; Cohen, M. L. Static Conductivity and Superconductivity of Carbon Nanotubes: Relations between Tubes and Sheets. Phys. Rev. B: Condens. Matter Mater. Phys. 1995, 52, 14935-14940.

(60) Vogt, P.; De Padova, P.; Quaresima, C.; Avila, J.; Frantzeskakis, E.; Asensio, M. C.; Resta, A.; Ealet, B.; Le Lay, G. Silicene: Compelling Experimental Evidence for Graphenelike Two-Dimensional Silicon. Phys. Rev. Lett. 2012, 108, 155501 .

(61) Cahangirov, S.; Topsakal, M.; Aktürk, E.; Şahin, H.; Ciraci, S. Two- and OneDimensional Honeycomb Structures of Silicon and Germanium. Phys. Rev. Lett. 2009, 102, 236804.

(62) Fleurence, A.; Friedlein, R.; Ozaki, T.; Kawai, H.; Wang, Y.; YamadaTakamura, Y. Experimental Evidence for Epitaxial Silicene on Diboride Thin Films. Phys. Rev. Lett. 2012, 108, 245501.

(63) Feng, B.; Ding, Z.; Meng, S.; Yao, Y.; He, X.; Cheng, P.; Chen, L.; Wu, K. Evidence of Silicene in Honeycomb Structures of Silicon on Ag (111). Nano Lett. 2012, 12, 3507-3511.

(64) Zhu, Z.; Tománek, D. Semiconducting Layered Blue Phosphorus: A Computational Study. Phys. Rev. Lett. 2014, 112, 176802 .

(65) Liu, H.; Neal, A. T.; Zhu, Z.; Luo, Z.; Xu, X.; Tománek, D.; Ye, P. D. Phosphorene: An Unexplored 2D Semiconductor with A High Hole Mobility. ACS Nano 2014, 8, 4033-4041.

(66) Gu, C.; Zhao, S.; Zhang, J. L.; Sun, S.; Yuan, K.; Hu, Z.; Han, C.; Ma, Z.; Wang, L.; Huo, F.; Huang, W.; Li, Z.; Chen, W. Growth of Quasi-FreeStanding Single-Layer Blue Phosphorus on Tellurium Monolayer Functionalized $\mathrm{Au}$ (111). ACS Nano 2017, 11, 49434949 .

(67) Zhang, J. L.; Zhao, S.; Han, C.; Wang, Z.; Zhong, S.; Sun, S.; Guo, R.; Zhou, X.; Gu, C. D.; Yuan, K. D. Epitaxial Growth of Single Layer Blue Phosphorus: A New Phase of Two-Dimensional Phosphorus. Nano Lett. 2016, 16, 4903-4908.

(68) Li, L.; Yu, Y.; Ye, G. J.; Ge, Q.; Ou, X.; Wu, H.; Feng, D.; Chen, X. H.; Zhang, Y. Black Phosphorus Field-Effect Transistors. Nat. Nanotechnol. 2014, 9, 372.

(69) Ji, D.; Cai, S.; Paudel, T. R.; Sun, H.; Zhang, C.; Han, L.; Wei, Y.; Zang, Y.; Gu, M.; Zhang, Y. Freestanding Crystalline Oxide Perovskites Down To the Monolayer Limit. Nature 2019, 570, 87. 